\title{
El estudio del árabe marroquí en España durante el siglo XIX. La obra de Pedro María del Castillo y Olivas, ¿árabe marroquí o árabe argelino?
}

\section{Moroccan Arabic or Algerian Arabic? The study of the Moroccan Arabic in Spain during the 19th. The book of Pedro María del Castillo y Olivas}

\author{
Francisco MOSCOSO GARCíA \\ Profesor titular Departamento de Estudios Árabes e Islámicos y Estudios Orientales \\ Universidad Autónoma de Madrid \\ francisco.moscoso@uam.es
}

Recibido: abril 2010

Aceptado: octubre 2010

\begin{abstract}
RESUMEN
La obra Diálogos españoles-árabes ó guía de la conversación mogharbi fue escrita en 1860 por Pedro María del Castillo y Olivas. Del análisis de la misma se desprende que el árabe en el que fue escrita es argelino y que se trata de un plagio de la obra de Delaporte, Guide, a pesar de estar dirigida a los militares españoles que ocuparon Tetuán tras la guerra hispano-marroquí. Su edición es reflejo del espíritu africanista que hubo en España durante el siglo XIX.
\end{abstract}

Palabras clave: Marruecos; Africanismo; Árabe marroquí; Árabe argelino; Siglo XIX.

\begin{abstract}
The book Diálogos españoles-árabes ó guía de la conversación mogharbi (Spanish-Arabic Dialogs or Moroccan Conversation Guide) was written in 1860 by Pedro María del Castillo y Olivas. Its study reflects that the Arabic used was Algerian and a plagiarism of the book Guide written by Delaporte, in spite of its aim to be addressed to the Spanish Military that occupied Tetouan after the SpanishMoroccan war. Its Edition is also a reflect of the Africanism Spirit that Spain lived during the $19^{\text {th }}$.
\end{abstract}

Keywords: Morocco; Africanism; Moroccan Arabic; Algerian Arabic; XIX century.

Índice: 1. Introducción, 1.1. Transcripción, 2. Análisis de los diálogos, 2.1. El nombre, 2.2. El verbo, 2.3. Adverbios, 2.4. Partículas, 3. Léxico de origen español, 4. Conclusiones, 5. Partes comparativas de las obras de Del Castillo (Diálogos) y Delaporte (Guide), 5.1. Índice, 5.2. Capítulo II (Diálogos) y I (Guide), 5.3. Capítulo XVI (Diálogos) y XIV (Guide), 5.4. Capítulo XXI (Diálogos) y XXII (Guide). 


\section{INTRODUCCIÓN}

Con este trabajo, pretendemos seguir contribuyendo al estudio del árabe marroquí durante el siglo XIX. Iniciamos nuestro recorrido por este período con el análisis del esbozo gramatical y el diccionario inédito escritos por Antonio Almagro Cárdenas en $1882^{1}$. Sólo fue publicada la primera parte en 1894 en las Actas del primer congreso de africanistas ${ }^{2}$. El segundo de los estudios que llevamos a cabo fue la descripción del viaje que Manuel Bacas Merino hizo a Marruecos entre 1798 y 1804 cuyo resultado dio a conocer al volver a España en 1807, con una gramática de árabe clásico en la que incluía, a modo de comparación, elementos fonéticos, morfológicos y sintácticos del árabe marroquí ${ }^{3}$. Gracias al viaje de este autor, tuvimos noticia de la existencia de una escuela de lengua árabe en Tánger, que funcionó entre 1800 y 1806. En ella se formó el franciscano Fray Martín del Rosario, que permaneció casi treinta años en Tánger haciendo labores de traducción e interpretación. Al parecer, escribió una gramática y un diccionario de árabe marroquí que nunca se publicaron y que se perdieron ${ }^{4}$. La segunda mitad del siglo estará marcada por la figura del padre Lerchundi, que es, sin lugar a dudas, si no el iniciador, sí el impulsor de los estudios de árabe marroquí en nuestro país. Sus dos obras, la Gramática y el Vocabulario, son el resultado de un trabajo de campo encomiable y unos conocimientos lingüísticos para lo que en aquella época había- bastante buenos. Probablemente su obra eclipsara los intentos posteriores de publicar nuevos trabajos. Almagro Cárdenas no llegó nunca a publicar su glosario y Del Castillo manifestó en el prólogo de sus Diálogos que quería publicar una gramática y un diccionario español-árabe, pero nunca vieron la luz ni tenemos noticia de que los manuscritos se encuentren en ninguna parte. En un trabajo que se publicará en el homenaje al P. Lourido, y que aún está en prensa, hemos analizado los préstamos al árabe marroquí de los romances peninsulares anteriores al nacimiento de España en el siglo XV y XVI y del español y otras lenguas de la Península en la etapa española ${ }^{5}$. La obra que pretendemos estudiar en esta ocasión es la que escribió Pedro María del Castillo y Olivas y fue publicada en 1860: Diálogos españoles-árabes ó guía de la conversación mogharbi. Madrid, Imprenta de M. Galiano, Plaza de los Ministerios, núm. 3. Para completar nuestro estudio sobre el estudio del árabe marroquí en España durante el siglo XIX, a la espera de que aparezcan nuevos datos, nos quedaría por nombrar a Juan Albino Tarsén y su libro Manual del lenguaje vulgar de los moros del Riff, publicado en Cádiz por la Imprenta de la Revista Médica en 1859. De esta obra nos ocuparemos en un próximo artículo que estamos preparando ${ }^{6}$.

${ }^{1}$ Cf. Rovira et al. 2002-2001.

${ }^{2}$ Cf. Almagro 1894: 85-108.

${ }^{3}$ Cf. Bacas 1807 y Moscoso 2008.

${ }^{4}$ Cf. Lourido 2002 y Castellanos 1898: 674-675.

${ }^{5} \mathrm{Cf}$. Moscoso 2010.

${ }^{6}$ Gómez (1996) da cuenta de la existencia de las obras de Del Castillo y Albino en su artículo. Albino anota en su libro que se trata de unos apuntes para uso particular que realizó en 1851 cuando estaba destinado en el Peñón de Gomera como militar. 
Nuevamente, nos encontramos ante la incomodidad de no saber mucho sobre la vida y la actividad laboral del autor, Pedro María del Castillo y Olivas en este caso. Manzanares de Cirre ${ }^{7}$ solo cita su libro en la bibliografía. Sabemos que perteneció a la "Sociedad Económica Matritense" " y que ocupó el cargo de secretario de la Sección de Agricultura, como se hace constar en la Guía de forasteros para el año de $1866^{\circ}$. Hemos estado personalmente consultando los ficheros en esta sociedad que se encuentra en Madrid, en la Plaza de la Villa, haciendo esquina con la calle del Codo, en la Torre de los Lujanes ${ }^{10}$. Pudimos consultar algunos escritos en los que figura el nombre de Del Castillo en calidad de secretario de la sección de agricultura, se trata de informes relacionados con esta actividad en relación a muestras o tratamientos. Nos explicaron que la sociedad solía funcionar en los siglos XVIII y XIX como una especie de consultoría para el Estado y otros estamentos en relación con estos asuntos. Con el número 437 y con fecha de 1864, aparece una ficha que lleva por nombre Sr. D. Pedro María del Castillo y Olivas y dentro un folio en el que se hace constar su admisión en la sociedad con fecha de 9 de enero de 1864. También, gracias a la portada de la obra, sabemos que era caballero de gracia ${ }^{11}$ de la ínclita orden militar de San Juan de Jerusalén ${ }^{12}$. Es todo lo que hemos podido saber de él.

Nuestro objetivo en el presente artículo va a ser únicamente la descripción del libro de Del Castillo y Olivas. Se trata de una obra que tiene 111 páginas y está dedicada -así lo hace constar en la portada- "al ejército de mar y tierra". En el prólogo de la obra, además de la ya mencionada intención de publicar una gramática y un diccionario, refiere que piratas marroquíes han atacado las costas españolas, motivo por el cual España entra en guerra y ocupa el territorio marroquí13

${ }^{7}$ Cf. Manzanares 1972.

${ }^{8}$ Real Sociedad Económica Matritense de amigos del país. Fudanda en 1775 por Carlos III. Actualmente todavía sigue existiendo: http://www.economicamatritense.com/

${ }^{9}$ Madrid, Imprenta Nacional, 1865.

${ }^{10}$ Agradecemos la amable acogida y la ayuda desinteresada que nos prestó la archivera, de quien sólo sabemos que se llama Fabiola.

${ }^{11}$ Así nombrado por el Gran Maestre de la Orden.

${ }^{12}$ Existieron tres grandes órdenes creadas a raíz de las cruzadas. La orden que sobrevivió más tiempo después de que el reino de Jerusalén fuera aniquilado por los mamelucos fue la de los Hospitalarios de San Juan de Jerusalén, creada en 1084. Esta orden nació como una hermandad de enfermeros para ocuparse de los enfermos. A principios del siglo XIV se instalaron en la isla de Rodas. Expulsados de esta isla por los turcos, Carlos V les ofrece en 1530 la isla de Malta para seguir haciéndoles frente. Tras la ocupación inglesa a principios del XIX, Malta dejó de ser su sede y se trasladan a Roma en 1834 (cf. Norwich 2008: 230 y ss. y 246 y ss.)

${ }^{13}$ No entendemos muy bien lo que quiere decir Del Castillo con 'piratas', probablemente mezcle acontecimientos: la presencia de piratas en el norte de África que atacaban a barcos españoles con los ataques en torno a Ceuta que desencadenaron el conflicto. Aunque por otro lado, durante el siglo XIX, se recurría a este justificación tal como consta en un artículo aparecido en El Museo Universal en su no 23, año III, 1 de diciembre de 1859: http:/www.cervantesvirtual.com/servlet/SirveObras/35761610103354729532279/203699 007.pdf. La confrontación entre España y Marruecos data de años atrás al inicio de la guerra en 1859. España había firmado un tratado con Marruecos en 1845 en el que se fijaban los límites fronterizos de Ceuta. Más tarde, el gobernador de esta ciudad inicia la construcción de una fortificación en terreno marroquí, 
El espíritu imperialista de nuestro país, en aquella época en gran decadencia, lo lleva a expresar el deseo de que la cruz sea puesta en los "minaretes de Fez y Mequinez". La guerra de África y la ocupación de Tetuán a comienzos de $1860^{14}$ son la justificación que el autor nos da para escribir su libro: "nuestros guerreros, nuestros empleados civiles y militares, nuestros sacerdotes y nuestros comerciantes, necesitan aprender el idioma mogharbi" (pp. 5-6). Como bien ha indicado López ${ }^{15}$, durante el gobierno del general O'Donnell y su partido La Unión Liberal, el espíritu patriótico y chovinista español, después de haber perdido muchas de sus colonias, cobra aliento, especialmente en el período que transcurre entre las dos revoluciones, la de 1854, conocida como 'La Vicalvarada', y la de 1868, 'La Gloriosa'. Es en este espíritu nacional en el que situamos la obra de nuestro autor, quien en la dedicatoria escribe: "Valientes guerreros!, Héroes de la Patria mía! Permitid que entusiasmado con vuestras proezas, os dedique este pequeño trabajo. Vuestro constante admirador, El autor" (p. 3).

Indudablemente, el libro, como él apunta en la portada, va dirigido sobre todo a los militares que participan en la guerra de África y que ocupan Tetuán durante tres años. El calificativo mogharbi "marroquí" y la toponimia a la que hace mención en los diferentes diálogos y de la que ponemos como ejemplos: Meknasa (Mequínez, p. 23), Suâra (Essaouria, p. 23), Thafflâ (Tafilal, p. 85), Tethauuan (Tetuán, p. 102),

incumpliendo España el tratado. Esto provocó la indignación de los marroquíes, quienes de noche destruyen lo que los españoles habían hecho y terminan por derribar "la piedra que marcaba el límite territorial". En 1859 también se firma otro tratado con el país vecino en relación a Melilla. Este mismo año, el cónsul español en Tánger dirige una carta al gobierno marroquí en el que le advierte de que el español no tolera "el ultraje inferido al pabellón español por las hordas salvajes que pueblan las provincias de Anghera" (cf. Alcalá 2005: 28-31). En agosto de 1859, nos dice Alcalá (2005: 39) que "la construcción de una casa fuerte, hecha levantar por el gobernador de Ceuta, en terreno neutral y a muy poca distancia de la plaza, fue la causa por la cual los marroquíes de Anghera hostilizaron las tropas encargadas de la vigilancia de los trabajos"; ésta será la causa por la que el 24 de octubre de 1859, España declare la guerra. O'Donnell veía en ello la justificación perfecta para afianzarse en el poder. Morales (2006: 185) nos dice también que Ceuta, Melilla, Chafarinas y los peñones venían sufriendo en la primera mitad del XIX ataques por parte de las tribus de Anghera, Guelaia, BeniUrriáguel y otras.

${ }^{14}$ Acerca de esta guerra que se desencadena en octubre de 1859 y tiene lugar durante la etapa de gobierno de la Unión Libral (1858-1863) presidida por Leopoldo O’Donell, cf. Alcalá 2005 y García Balañà 2002.

${ }^{15}$ 2008: 107. Más adelante (p. 109), dice que "la guerra de 1859 tiene el aliciente histórico de la lucha contra el moro infiel, tema que el nuevo nacionalismo redescubre en la historia”. Fruto y aliento al mismo tiempo de este patriotismo son las obras de Alarcón y Núñez de Arce, cronistas que se encuentran presentes en el campo de batalla. La obra de Alarcón, según López, tiene "un componente patriótico" bastante bien marcado que convierte "cualquier rasgo de maurofilia en una simple referencia pintoresca sin la mayor relevancia y sin peso decisivo en el libro" (p. 106), y es "el ejemplo más evidente de literatura al servicio del poder y, en concreto, al servicio de la política de la Unión Liberal" (p. 148).

${ }^{16} \mathrm{La} O$ indica labialización en contacto con $\dot{g}$, rasgo típico de los dialectos de tipo beduino y presente en algunos sedentarios de la costa atlántica. Sobre esto, cf. Colin, "al-Maghrib", $E I^{2}$, vol. V (“Aperçu linguistique"), p. 1194. Pueden labializarse también las pospalatales $k$ y $g$, las velares $x, \dot{g}$ y $q$ y las labiales $b$ y $m$. 
Tandjia (Tánger, p. 107), Faz (Fez, p. 110) son prueba de que Del Castillo dirige su mirada hacia Marruecos. Con respecto al adjetivo "marroquí" al que hacíamos alusión anteriormente, debemos trasladarnos a los Diálogos y a su análisis lingüístico para darnos cuenta de que el registro empleado no es el marroquí sino el argelino. En la fecha en la que publicó la obra, Francia ya llevaba treinta años ocupando el norte de Argelia. Sólo podemos arrojar en un primer momento dos hipótesis después del análisis de los textos y la carencia de información sobre la vida del autor: o que tuviera un informante argelino o que viajara a Argel o a París en donde pudo estudiar con los manuales de árabe argelino que ya en la época habían sido publicados. Entre estos, destacamos los siguientes:

ROLAND DE BUSSY, Th. L'idiome d'Alger ou dictionnaires français-arabe et arabe français précédés des principes grammaticaux de cette langue par T. Roland de Bussy, directeur de l'Imprimerie du Gouvernement. Alger: Brachet et Bastide Libraires-éditeurs, Place du Gouvernement, 1838.

DELAPORTE, J. Honorat. Guide de la conversation français-arabe, ou dialogues en arabe et en français, avec le mot à mot et la prononciation interlinéaire figurés en caractères français, corrigés et augmentés par J. Honorat Delaporte, secrétaire-interprète de la Direction de l'Intérieur en Algérie. $3^{\text {ème }}$ édition. Alger, 1846.

DELAPORTE, J. Honorat. Principes de l'idiome arabe en usage à Alger, suivis de phrases familières et d'un conte arabe, avec la prononciation et le mot à mot interlinéaires par J. H. Delaporte fils. Alger, 1845.

CHERBONNEAU, Auguste. Dialogues arabes à l'usage des fonctionnaires et des employés de l'Algérie. Alger: Dubos frères, 1858.

A estas obras, habría que añadir los dos trabajos sobre árabe argelino que Cherbonneau ${ }^{17}$ publicara en la revista Journal Asiatique, y que se detallan en la Bibliografía. Por otro lado, es probable que Del Castillo contara con alguna otra obra más de la que no hemos tenido noticia, quizás los trabajos que más abajo detalla Cherbonneau. Hemos podido consultar las cuatro obras citadas anteriormente. En una primera hojeada, nos llamó la atención el parecido que tiene con la segunda de ellas. Delaporte ofrece en su libro treinta y nueve diálogos y Del Castillo treinta. Tras una lectura atenta, pudimos comprobar que los diálogos de Del Castillo habían sido tomados íntegramente de los de Delaporte, tanto el título como las expresiones, con alguna variante, sobre todo en lo relacionado con los topónimos. Al final de este artículo, incluimos tres páginas de los Diálogos y las partes correspondientes en Guide para que el lector compruebe directamente el plagio. El esquema que sigue Delaporte en su Guide y Cherbonneau en sus

${ }^{17}$ Sobre este autor sabemos lo que se nos dice en la portada de sus obras, en Dialogues se nos dice: " Professeur d'arabe à la chaire de Constantine ; Correspondant du Ministère de l'Instruction publique et des Cultes, pour les travaux historiques; Membre de la Société asiatique ; Secrétaire de la Société archéologique de la province de Constantine ". Y en el Dictionnaire lo siguiente : "Correspondant de l'Institut, ancien directeur du Collège Arabe-français d'Alger". 
Dialogues es el mismo: primero da el texto en francés y luego en árabe argelino (en grafía árabe y en transcripción) seguido de una transcripción literal. Es lo mismo que hace Del Castillo, aunque él no incluye la grafía árabe, probablemente por motivos de imprenta, o probablemente no conociera la grafía árabe. En cualquier caso, podemos suponer que Del Castillo estuviese en Argel siguiendo los cursos que Delaporte daba o simplemente se hiciera con su libro en cualquier parte. Cherbonneau, en la introducción de su obra, afirma: "Déjà MM. Delaporte, chef du bureau arabe départemental à Alger, et Martin, interprète principal, avaient publié avant 1840 des manuels de conversation, appropriés aux besoins de l'armée et des colons. Notre livre, bien que nouveau quant à sa véritable destination, peut être considéré comme faisant suite aux travaux de ces orientalistes distingués. Nous nous adressons plus spécialement aux administrateurs, lesquels ont pour mission de faire connaitre aux musulmans les intentions bienveillantes et toutes paternelles du gouvernement français" (p. II). Los temas de la obra Dialogues son-según el autor- "L'agriculture et l'économie domestique nous ont paru les meilleurs sujets à traiter [...] les musulmans de l'Algérie apprendront, sans effort de raisonnement, le mécanisme de notre système administratif..." (p. I).

Es probable que Del Castillo eligiera mogharbi por dos motivos, el primero por el espíritu patriótico español que dirigía su mirada hacia el norte de Marruecos y el segundo porque era consciente de que, aunque el librillo fuera dirigido a militares españoles en el norte de Marruecos, el árabe que refleja se parecía bastante al de esta zona por pertenecer a la misma familia del árabe hablado en la región del Magreb ${ }^{18}$. A esto debemos añadir en palabras de Morales (2006: 181) que "el africanismo español de inclinación marroquinista hizo en el siglo XIX un ensayo general previo de corte argelino", ya que Francia había conquistado la regencia de Argel en 1830, hecho que provocó un gran interés entre esta fecha y 1850 en nuestro país, especialmente en el seno de los militares, como queda reflejado en una serie de obras sobre Argelia de intereses diversos: "militares y geopolíticos... geográficos y costumbristas" (Morales 2006: 18). Se trataba de "un argelianismo español frustrado", de inspiración oranesa, otrora española, que alentó el africanismo marroquinista. Si bien no sabemos mucho de Del Castillo, sin ninguna duda, hay que situar su obra en este contexto histórico y, como ya hemos sugerido anteriormente, es muy probable que hiciera un viaje a Argel acompañando a alguna delegación.

El librillo comienza con una explicación de la transcripción que va a emplear y está dividido en treinta "diálogos" con frases empleadas en determinados contextos comunicativos como "saludar y cumplimentar" (II), "para una marcha militar (IV), "del tiempo, de las estaciones, del aire, etc." (VIII), "en el café" (XV), "para tomar informes a alguna persona" (XXI), "para tomar un baño moro" (XXVI) o "para hablar con un zapatero" (XXX). Cada diálogo está dividido en tres columnas: en la primera se nos da la traducción literaria al español, en la segunda se presenta el texto en árabe y en la tercera se nos da una traducción literal.

\footnotetext{
${ }^{18}$ Para tener una visión de conjunto de los dialectos árabes hablado en esta zona, cf. Marçais 1977.
} 


\subsection{TRANSCRIPCIÓN}

La transcripción (pp. 7-11) que nos ofrece Del Castillo es la misma que aparece en los trabajos de Delaporte, Cherbonneau y Roland De Bussy, con alguna salvedad. La del primero es idéntica a la Del Castillo, sólo que para $h$ Delaporte transcribe $h h$, para $\dot{g} g h$ y para $s s s$ mientras que Del Castillo emplea $h, r r$ y $c$ respectivamente. Cherbonneau y Roland De Bussy emplean para $\dot{g} r$ y para las enfáticas, el primero un punto debajo de la letra y el segundo hace como Del Castillo, es decir, acompañar la grafía de $h$. El autor de los Diálogos no menciona en ningún momento los rasgos típicos de la fonética del árabe hablado en el norte de Marruecos. Entre las características más destacadas de su presentación de las letras están:

1.1.1 Nos advierte que la $p$ (oclusiva bilabial sorda) no existe en el alfabeto árabe y que los "nombres extranjeros" se escriben con su correspondiente sonora $b$. Ejemplo: bedru "Pedro".

1.1.2. Para $t$ (oclusiva dental sorda), de la que dice que se pronuncia como la del español, no da la variante africada [ț]. Pero luego, siguiendo el orden alfabético del árabe clásico, dice que está la letra $t s$, de la que comenta: "una letra compuesta de dos sonidos, la $t$ y la $s$ ”. Se trata de la $ث$ del árabe clásico (interdental), que no existe en árabe marroquí y que se pronuncia en esta lengua con la realización [ț] de $t$; en algunos casos, en la región de Yebala, podemos encontrar la variante [t] (interdental) cuando $t$ aparece en posición intervocálica o posvocálica en final de palabra ${ }^{19}$, fenómeno conocido como fricatización. Ejemplos: ech-chetsa (ə̌̌-šta) "la lluvia" (40), sâatsein (sā ātēyn) "dos horas" (43), qahutsek (qhütək) "tu café” (51).

1.1.3. La $z \check{z}$ está transcrita como $d j$. Dice que se pronuncia como la $j$ francesa pero "va siempre unida á la de la $d$ que precede siempre". En Marruecos, el fonema es $\check{z}$ (fricativa prepalatal sonora) con su variante en la zona norte [ $\breve{g}]$ (africada) en posición geminada ${ }^{20}$. Djit (žìt) "he venido".

1.1.4. Distingue entre $d$ (oclusiva dental sonora) y $d z$ (fricativa interdental), aunque no se entiende muy bien lo que quiere decir: "letra compuesta por dos sonidos, d y z, esta última con pronunciación francesa" (p. 8). Los ejemplos que pone no son muy aclaratorios: $d z a q$ (dāq. ác. $\underline{d} \bar{a} q$. DFA $\underline{d} \bar{a} q$ en goutter) "paladear" y añade "pronúnciase ed-dsaq", dzial (dyā̄l. DFA dyāl en de con dental) "de mí a mí; pronúnciase ed-sial". En árabe marroquí no existe esta última letra como fonema. Es cierto que en el norte, al igual que [t] , puede aparecer [d] en el mismo contexto (cf. $\S$ 1.2. y la nota correspondiente) $)^{21}$.

${ }^{19}$ Cf. Moscoso 2003: 72 y la biliografía propuesta. Se trata de un rasgo típico del rifeño y sustrato o adstrato en el árabe que se habla en la región de Yebala. Este fenómeno se extiende a las otras dentales, $d$ y $d$, a la labial $b$ y a las palatales $k$ y $g$.

${ }^{20}$ Sobre $\check{z}$ en el norte de África y el alófono [ $\breve{g}$ ], cf. Cantineau 1960: 57-59. Cantineau (1937) dice que el grupo $\mathrm{B}$ (parler des nómades telliens) tiene $\breve{g}(=d j)$. Este tipo pertenece a nómadas sedentarizados. Cantineau también indica la presencia de dialectos sedentarios en Argel, Blida, Cherchell, Dellys, Médéa, Miliana y Ténès, fuertemente influenciados por los del grupo B (Cantineau 1937: 8).

${ }^{21}$ Es muy probable que esté describiendo la presencia de interdentales correspondientes a los dialectos beduinos de Argelia (cf. Cantineau 1937). 
1.1.5. Los fonemas velarizados dentales están indicados con un dígrafo compuesto por las grafías latinas haciéndoles seguir de $h$, es decir $d h=d$ (oclusiva dental sonora velarizada), $t h=t$ (oclusiva dental sorda velarizada). Para $\underline{d}$ (fricativa dental sorda velarizada), que tampoco existe en árabe marroquí sino como alófono de $d$ en el norte y en los mismos contextos indicados para [t] y [ㅁ] , emplea la misma transcripción que para $d, t h$, y dice que "la pronunciación se confunde con la anterior".

1.1.6. La $q$ se realiza como [q] (oclusiva velar sorda), de la que dice que su realización está "entre $c a, c u$ y $g a, g u$, esto es, entre la $q$ y la $g$ suave" 22 .

1.1.7. En cuanto al vocalismo, habla de tres vocales largas, $a, u$ e $i(\bar{a}, \bar{e}, \bar{\imath}) \mathrm{y}$ tres breves, $e, i$ y $u$ y advierte que "muchas veces" la $e$ se pronuncia $o^{23}$.

1.1.8. Se señalan sílabas abiertas que no aparecen en árabe marroquíi ${ }^{24}$. Ejemplo: tementach (tmənțās) "dieciocho". También vocales de apoyo al iniciar algunas voces que tampoco se oyen. Ejemplos: etnin u achrin (tnīn u Sašrīn) "veintiuno".

\section{ANÁLISIS DE LOS DIÁLOGOS}

Pretendemos con este análisis lingüístico, hacer ver que el árabe en el que se basó el autor no pertenece al de la región de Yebala ni a las zonas centro y sur, aunque esto está suficientemente demostrado desde el momento en el que descubrimos que su obra es un plagio, sino que se trata de árabe argelino, el empleado en Argel, exactamente el mismo que recoge Delaporte, Roland De Bussy

${ }^{22} \mathrm{La}$ diferencia entre dialectos prehilalíes y hilalíes en el Magreb está, groso modo, aunque también habría que incluir otros rasgos, en que los primeros pronuncian $q$ y los segundos su correspondiente sonoro $g$. En el caso de Marruecos y en el norte, se trataría del primero de los casos, aunque en árabe chauní y teuaní antiguo, también se da $?$ (oclusivo glotal sordo). Sobre esto, véase la bibliografía y las explicaciones que se ofrecen en Moscoso 2003: 45-48. Cantineau (1937: 3-4) dice que en Argelia, los dialectos de Constantina son principalmente prehilalíes (cf. Cantineau 1938-1939: 5), mientras que para el conjunto del país, de manera general, se puede afirmar que los dialectos son del segundo tipo (B), lo cual se pone de manifiesto en el departamento de Argel.

${ }^{23}$ No parece definir la situación del vocalismo, especialmente en lo relacionado con las vocales breves. El sistema vocálico del norte y centro de Marruecos estaría formado por el binomio $ə \neq \breve{u}$. La $e, i$, $u$ y $o$, a la que podríamos añadir $a$ serían variantes reducidas de otros tantos alófonos vocálicos cuya coloración depende del contexto consonántico en el que se encuentre. El hecho de que aparezca $e$ e $i$, es quizás reflejo de que en el norte estos dos alófonos de a pueden aparecer en los mismos contextos (Moscoso 2003: 31). Acerca del vocalismo en árabe marroquí, cf. Aguadé 2008: 290-292, quien habla de tres sistemas vocálicos: el primero, "el más extendido, tanto en dialectos hilalíes como prehilalíes", y que es la base del árabe marroquí estándar, se basa en tres vocales largas $(\bar{a}, \bar{l} \mathrm{y} \bar{u}) \mathrm{y}$ dos breves $(\partial \mathrm{y} u)$. El segundo aparece en dialectos hilalíes del noreste de Marruecos y está basado en las mismas vocales largas $\mathrm{y}$ en tres breves $(a, \partial \mathrm{y} u)$. Y el tercero se corresponde con el del hassānyya, que se habla en el el Sáhara marroquí, Mauritania y suroeste argelino, el cual está formado por tres vocales largas $(\bar{a}, \bar{l} \mathrm{y} \bar{u})$ y dos subgrupos en las breves ( $a, i$ y $u$ en sílabas abiertas y ə y $a$ en sílabas cerradas).

${ }^{24}$ Sobre la inexistencia de sílabas abiertas en árabe marroquí, al contrario que en árabe clásico, y la estructura silábica, cf. Moscoso 2004: 20. 
y Cherbonneau ${ }^{25}$. La carencia de una buena transcripción y otros rasgos morfológicos relevantes, nos ha obligado a ofrecer una visión de conjunto, pero creemos que acertada en cuanto a la relevancia de los rasgos seleccionados. Incluimos la traducción que el autor nos ofrece y, en algún caso, hemos anotado la nuestra cuando pensamos que podría haberse mejorado.

\subsection{EL NOMBRE:}

- anaia (ānāya) “yo" (p. 30), ntaia (ntāya) (p. 102).

- butellis "pesadilla" (p. 15). (DFA p. 53).

- etchina (čina) "naranjas" (p. 94).

- mleh (mlīh) "bien, bueno" (p. 55). Rahi mleha bezzaf (rāhi mlīha bəzzāf) "está muy buena" (p. 56).

- qus en-nabi (qūs en-nābi) "arcoíris" (p. 36). (DFA p. 25).

- rrâchi (g்āši) "populacho" (p. 24).

- sibiqlia "Sevilla" (p. 86).

- çaha âlik (ş̧hha Slīk) "quedo agradecido" (p. 94).

- sbaniulia, françiça, enqliza, thaliana "española, francesa, inglesa, italiana" (p. 85).

- sbaniul, françiç, enqliz, thalian "español, francés, inglés, italiano" (gentilicios) (p. 85). es-sbaniuli “el español” (gentilicio) (p. 91).

- tesâ (təs $\{a)$ "nueve" (p. 13). (DFA p. 367) En árabe marroquí tes $\{\bar{u} d$.

\subsection{EL VERBO:}

- El preverbio es inexistente. No lo recoge ni Cherbonneau ni Roland De Bussy. En árabe marroquí suele ser $k \bar{a}-\mathrm{o} t \bar{a}-$, y en la región de Yebala podemos oír $l \bar{a}-\mathrm{e}$ incluso la forma flexionada, documentada en Chefchaouen, $n \bar{a}-, d \bar{a}-, l \bar{a}-$.

- Para la segunda persona masculina singular del perfectivo, el sufijo es - $t$. Ejemplo: bedit (bdīt) (enta) "tú has empezado" (pp. 100 y 101). Aunque Del Castillo no recoge la forma femenina, ésta sería bdīti tal como se recoge en Roland De Bussy (p. 40).

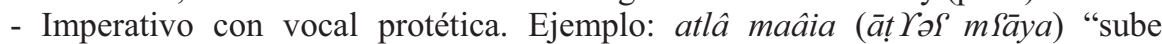
conmigo" (p. 69). La vocal protética se recoge para verbos sanos, defectivos y asimilados en Roland De Bussy (p. 35 y ss.)

Los verbos que a continuación se recogen suelen ser típicamente argelinos y se recogen en las obras de Cherbonneau y Roland De Bussy.

- ( $\dot{g} l \partial q)$ errleq eth-thuaqi ( $\dot{g} l \partial q$ ot-twāqi) "cierra las ventanas" (p. 60). merrluq bel-meftah (məg்lüq b ol-məftāh) "cerrado con llave" (p. 47).

- (həbb) habu (həbbu) "ellos quieren" (p. 25), rak, thebb, tedhhak? (rāk thəbb tədhək?) "¿te quieres reír?” (p. 31). thebb chi chuiia merqa? (thəbb ši šwəyya mərqa?) "quieres un poco de caldo" (p. 56).

${ }^{25}$ Destacamos algunos datos, a modo de muestra, que coinciden con los rasgos de la obra de Cherbonneau: emmâla "entonces" (p. 2), ma da byya "desearía" (p. 5), ra-houm qarraboue "están cerca de" (p. 5), soua soua "exactamente” (p. 8), qaddache? "¿cuántos?” (p. 12), enta ya "tú" (p. 13), mta" 'a "de" (posesión) (p. 21), a lache? "¿por qué?” (p. 36), iheubb "él quiere” (p. 37), ta amel hakda "él hace así" (p. 45), iza "si" (p. 47 partícula ìda), derouokt "ahora" (p. 58), delouokt "ahora" (p. 65), mlih "bueno, bien" (p. 77), oueïna? "¿dónde?" (p. 100). 
- (nžəm) nendjem (nənžəm) "yo puedo" (p. 16). (DFA p. 440).

- (qəbb) enqebb (nqəbb) "yo quiero" (p. 18). Creemos que se trata de una errata. $q ə b b$ no aparece en $D F A$.

- (q९əd) aqâd chuiia (q§əd šwiyya) "siéntate un poco" (p. 17).

- (rāh) ruh (rūh) "ve" (p. 25).

- (səlləm) allah isellmek (ərYāh ys əllmək) "gracias" (p. 80).

- (şbb) rahi tçebb ech-chta (rāhi tșəbb əš-šta) "llueve" (p. 36).

- (Sməl) tâmel fiia khir kebir (təSmol fəyya xīr kbīr) "me harías un gran servicio" (p. 26). âmel eth-thrumbâ (Smol eț-țronba) "toma algunas lavativas" (p. 75).

\subsection{ADVERBIOS}

Al igual que los verbos anteriores, estos adverbios son típicamente argelinos y se recogen en las obras citadas anteriormente.

- delueqt (dolwoqt) "ahora" (p. 40).

- iruh emmala el-ium? (yrūh əmmāla ol-lyūm?) “¿sale, pues, hoy?” (p. 76).

- kach ysafru? (kāš ysāfru?) “¿cómo viajan?” (p. 82).

- mazelt ma amentek (māzolt ma āmontək) "me cuesta trabajo creerte" ("todavía no te creo") (p. 30).

- rredua (gədwa) "mañana" (p. 16). Pero también rredda (ġdda) "mañana" (p. 74). Las dos variantes se recogen en $D F A$.

- sua sua "exactamente" (p. 86).

\subsection{PARTÍCULAS}

Igualmente, estas partículas forman parte de las particularidades del árabe argelino y se recogen en las dos obras anteriormente citadas.

- (mādābəyya) Ma da bia nendjem nuasi lek emziia khir men hadi (mādābəyya nənžəm nwașși lək mzəyya xīr mən hādi) "desearía hacerte mayor servicio" (p. 21) (desearía ofrecerte un mejor servicio que éste). ma-da-bia en-chereb uahed el-kas mtâ el urdjatha berdha (mādābəyya nəšŗəb wāḥ̂ əl-kās mtâ̄ el-əŗžāṭa băŗda) "me gustaría beber una horchata fresca" (p. 67).

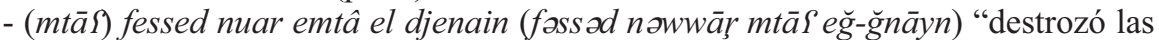
flores de los jardines" (p. 39). Chuiia mtâ el-leham (šwəyya mtâ̊ əl-lhəm) "un poco de carne" (51). El qlûma mtaâk (əl-qlüma mtâ̧ək) "tus plumas" (p. 76). Aunque en un caso, hemos encontrado dyāl: el kuskus dialek mleh bezzaf (al-kaskas dyālek mlīh bəzzāf) "tu cuscús está muy bueno" (p. 56). Las dos partículas se recogen en DFA y en la gramática de Roland De Bussy (p. 29).

- (ra) El uso continuado de la partícula ra + pronombre personal sufijado para expresar el estado de ánimo. Ejemplos: Fain rak machi? (fāyn ŗāk māši?) "¿a dónde vas?" (p. 23), el-ârab ma rahum chi emkhabiin feththriq? (əl-\{̧rab ma ŗāhəm ši mxəbbyīn $f$ at-trīq?) "¿los árabes no están escondidos en el camino?” (p. 24), rak tetkellem beç-çah? (rāk tətkalləm b əș-ṣəhh?) “¿hablas con seriedad?” (p. 30), Rani ferhan bezzaf (rāni fərhhān bəzzäf) "estoy muy alegre" (p. 33), er-rih rah bard (ər-rīh rāh bərd) "el viento es frío" (p. 37).

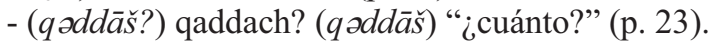

- uain chachiitsi? (wəyn šyyšîti?) “¿dónde está mi gorro?” (p. 99). 


\section{LÉXICO DE ORIGEN ESPAÑOL}

En el análisis de estas voces, anotamos después de cada una lo que dice el autor en relación a su origen, en caso de que así lo hiciera, y luego lo dicho en el estudio que ya hemos hecho sobre el Vocabulario del P. Lerchundi, y la información que hemos encontrado en el diccionario de Prémare $(D A F)$, el de Beaussier, de Cherbonneau y de Roland De Bussy.

- biçetha (bīṣeța) “peseta” (p. 109). Cf. bassīṭa en Moscoso 2010 (esp.)

- beracheka (bərrəška) “tempestad” (p. 35). Cf. bărrāška en Moscoso 2010 (esp. s. XVI "barrusca”) y Beaussier.

- biskutchu (biskutšu) "bollos” (p. 58). Cf. b̌̌škōto en Moscoso 2010 (probablemente su origen es la lingua franca y del italiano biscotto).

- dūru-bu-medfâ (dūru bū-mədfər) "duro columnario" (p. 95) (así llaman a nuestros duros columnarios), "veinte reales" (p. 109). Cf. dūru en Moscoso 2010 (esp. "duro"). Beaussier llama al dūru bü-mədfəS "piastra de España" cuyo valor es de cinco feluses con cuarenta.

- el-faltha (ol-fälța) "la falta" (p. 21); Del Castillo dice que es española e "importada" por los moriscos". Cf. Moscoso 2010 (lingua franca y del esp. "falta") y Beaussier.

- garfuiat (gərfūyāt) "tenedores" (p. 51). DAF 10: 682 (esp. "garfio"). DFA.

- lazem lek takel chuiia bulqâ (lāzəm lək tākəl šwəyya bulqa) "necesitas purgarte" (p. 74); dice que es una palabra española, y aunque no lo dice, le debe sonar a "purgar".

- lesun (lēsūn) "lección” (p. 85). Esta voz es sin lugar a dudas francesa, leçon, lo cual nos ofrece una prueba de que el informante podría ser argelino.

- matrit (mətrīt) "Madrid" (p. 39). Cf. mādrīd y măğrīt (inus.) en Moscoso 2010.

- muda (mūda) (p. 70) "moda, costumbre"; dice que es de origen español. Cf. mōda en Moscoso 2010 (esp. "moda").

- qalma (kālma) "calma” (p. 37); de origen español según el autor. Cf. kălma "calma en el mar, bonanza" en Moscoso 2010 (esp. "calma")

- el-qirra (ol-girra) "la guerra". Dice que es de origen español. Cf. DAF 10: 751 (esp. "guerra")

- qusthu (gusțo) "gusto" (p. 26) de la que también dice lo mismo que la anterior. Cf. güšțo en Moscoso 2010 (esp. en la lengua franca gusto).

- siqaru (sīgāron "cigarro" (p. 67). Cf. Beaussier.

- siquru (sīqūru) "seguro" (p. 41); literalmente dice "voz española usada en las poblaciones". Beaussier recoge esta voz pero como adverbio y nos da el significado "seguramente", advirtiendo entre paréntesis que procede del español.

- suma (süma) "suma, coste" (p. 70). Cf. Beaussier.

- thrumbâ (țronba) "lavativa" (p. 75). Dice que es de origen italiano. En Moscoso 2010 tenemos ot-trŏnba '(el) trompo (con el que juegan los muchachos)' (esp cat tromba). Cf. también Beaussier, quien ofrece el sentido que da Del Castillo. 


\section{CONCLUSIONES}

Poco hemos podido saber sobre la vida de Del Castillo, no más de lo que él anota en la portada de su libro y su actividad en la Sociedad Económica Matritense. Lo más destacado de su obra es que está publicada en un contexto en el que el africanismo español de orientación marroquinista es palpable en la sociedad, tal como demuestra el que dedicara los Diálogos a los militares que ocupan la ciudad de Tetuán. Por otro lado, el estudio lingüístico de los textos nos lleva a concluir que el árabe en el que está escrito es argelino, en concreto el hablado en Argel. Este dato es corroborado por la similitud con la obra de Delaporte (Guide) que ha plagiado casi en su totalidad. Y además, la Historia nos demuestra cómo entre 1830, fecha en la que Francia ocupa la regencia de Argel, y 1850, se escriben en España una serie de obras sobre Argelia en las que el espíritu africanista se inspira para dirigir su mirada hacia Marruecos.

\section{PARTES COMPARATIVAS DE LAS OBRAS DE DEL CASTILLO (DIÁLOGOS) Y DELAPORTE (GUIDE)}

En este apartado, a modo de apéndice, hemos incluido algunas partes de la obra de Del Castillo comparándolas con otras de Delaporte para que comprobemos directamente el plagio.

\section{1. ÍNDICE}

\section{Diálogos:}

\section{INDICE.}

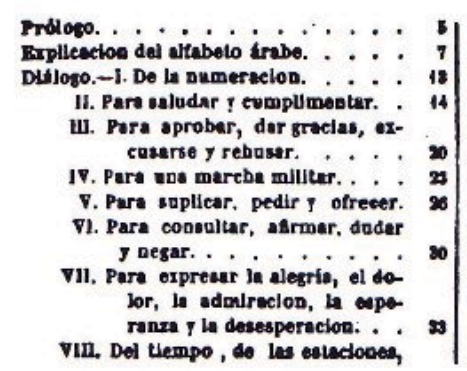

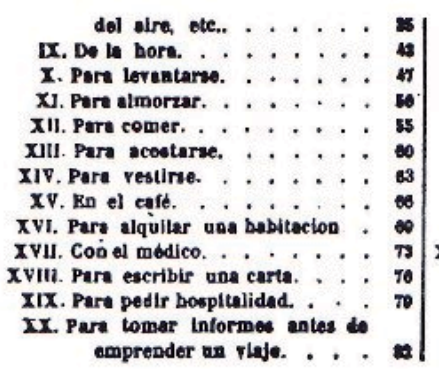

XX. Park tomar loformes it alsan persone. . . . . . . XXII. Pare pedtr botleies varles. . . XXIII. Para is a cazar. . , , , , $\infty$ XxIV. Del paseo. . . . . . . . . XXV. Pare comprar un caballo. . . w XXVI Pare tomar un beño moro. . XXVII. Para el estudio de la lengua ofpañole. . . . . . . 100

XXVIII. Para admitir on crlado. . . 100 XXIX. Para alqullar, 6 comprar abe casa de campa. . . . . . . 104

XXX. Para hablar con en expatero: 100 


\section{Guide:}

\section{TABLE DES MATIERES.}

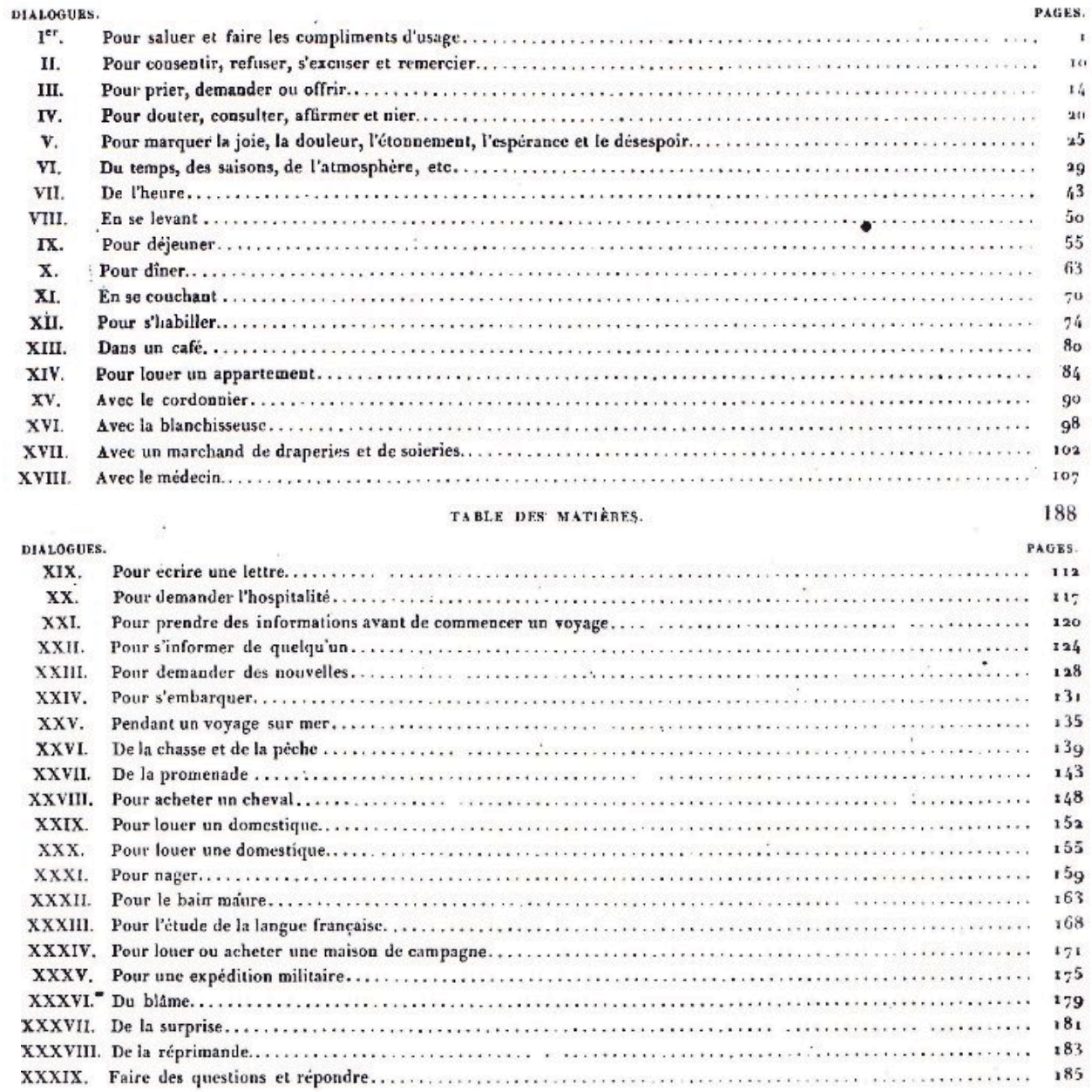




\subsection{CAPÍTULO II (DIÁLOGOS) Y I (GUIDE)}

\section{Diálogos}

\section{DIALOGO II. \\ Para saludar, eumplimentar y dar gracias.}

Buenos dias, caballero.

Te saludo.

Te saludo á mi vez.

Hace mucho tiempo que no tengo el gusto de verte.

Cómo estás?

Bien, gracias á Dios.

Me alegro de que estés bueno.

Y tú, cómo estás?

No muy bien, estoy algo indispuesto.

El calor me atormenta.

Sin embargo, el viento del desierto no

se siente hoy.

Que el dia te sea dichoso.

Te beso las manos.

$\mathrm{Y}$ en tu casa , cómo siguen?

Bion, que Dios te quiera.
Çbah el kher ia sidi. Selam allaik.

Âlaik es-selam.

Qaddach ma chuf-tek chi.

Ach enta ?

Bekher el khamdu allah.

Rani ferham belli enta bekher.

U enta, u ach halek?

Ma fi hali chi rani chuiia mridh.

E8-sekhวna rrammetni.

Kif-ach el qabli ma ikhedem chi el-ium.

Enharek mebruk.

Rani enbus bieddek.

Kach rahi darek?

Bekber, allah iazzek.
Mañana la buena 6 mi señor.

Salud sobre ti.

Sobre tí la salud.

Cuánto no visto $k$ ts.

Cómo tú?

Bien las alabanzas á Dios.

Yo soy contento que tá bueno.

Y tú, y cómo estado tuyo?

No en estado mio, yo soy un poco enfermo.

El calor ahoga á mi.

Como que el siroco no ha trabajado hoy.

Dia tuyo dichoso.

Yo soy yo beso con mano tura.

Cómo es casa tuya ? -

Con bien, Dios quiera á tr.

\section{Guide}

\section{DIALOGUES FRANCQAIS-ARABES}

\section{DIALOGUE PREMIER.}

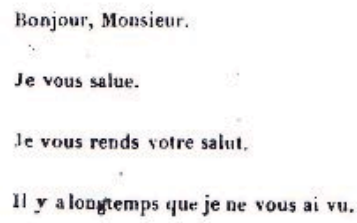

POUR SALUBR BI PAIRB LRS COMPLIMETTS D'TSAGE.

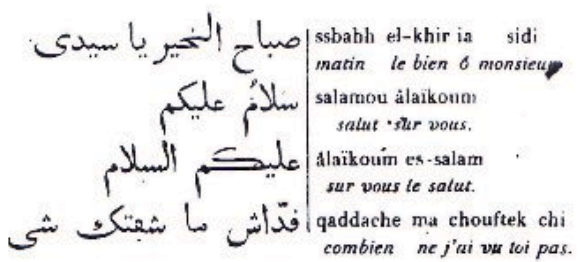


Comment vous portez-vous?

Bien, grâces à Dieu.

Je suís charmé de vous voir en bonne santé.

Et vous, comment vous portez-vous ?

Pas très-bien, je suis un peu malade.

La chaleur m'ètoufie.

Comment! le vent du désert ne souffle pas aujourd'hui.

Que votre jour soit heureux.

Je vous baise les mains.

Comment se porte-t-on chez vous?
DALOGUE 1.

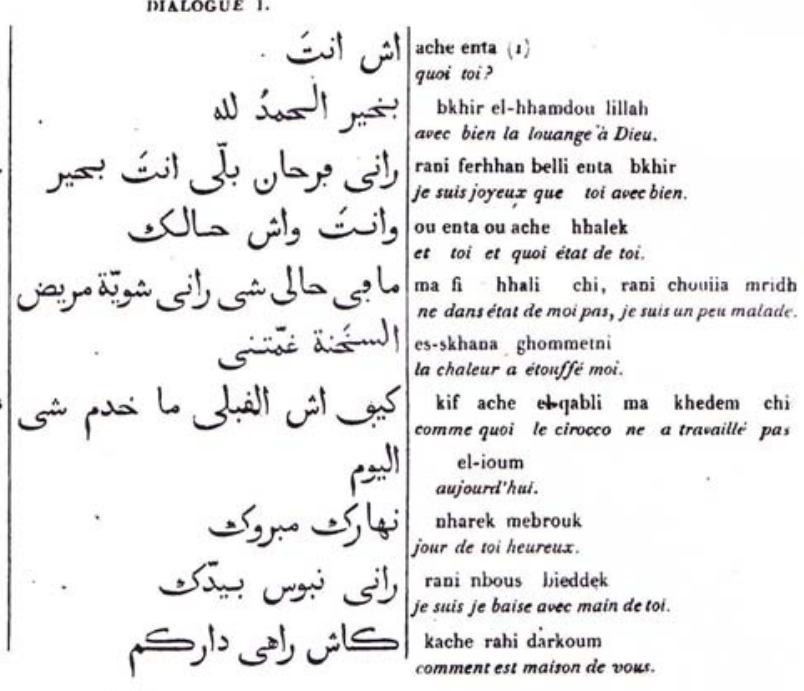

(1) On dit aussi entsa; le se prononce tantòt comnie un t, tantỏt comme $t s$

\subsection{CAPÍTULO XVI (DIÁLOGOS) Y XIV (GUIDE)}

\section{Diálogos}

\section{DIALOGO XVI:}

\section{Para al quilar una habitaclom.}

\begin{tabular}{|c|c|c|}
\hline Bres tá el duoĩo de esta cass? & Ente mula had ed-dar? & Tu dueño esta la casa? \\
\hline Sí señor. & Eh, ia sidi. & Sl, olı mi señor. \\
\hline $\begin{array}{l}\text { Tienes para alquilar alguna habita- } \\
\text { cinn? }\end{array}$ & Andek chi mesken (1) letkra? & Tienes tú habitacion en alquiler? \\
\hline Tengo une muy bonita en la axotea. & $\begin{array}{l}\text { Andi uahed ol mensah (2) mleh fuq } \\
\text { esthah. }\end{array}$ & $\begin{array}{l}\text { Tengo una buena habitacion arriba } \\
\text { el azotea. }\end{array}$ \\
\hline $\begin{array}{l}\text { No teadrias une situede on ol vo } \\
\text { randah? }\end{array}$ & $\begin{array}{l}\text { Ma andek chi uahed el rrerfa fel- } \\
\text { fuqani? }\end{array}$ & $\begin{array}{l}\text { No tienes una babitacion en el prin- } \\
\text { cipal? }\end{array}$ \\
\hline $\begin{array}{l}\text { No tengo en este momento. } \\
\text { E.sséñame la haluitacion de la asoten. }\end{array}$ & $\begin{array}{l}\text { Ma andi chi ti had es-sáa. } \\
\text { Urri li el-menzah elli fuq es-bthah. }\end{array}$ & $\begin{array}{l}\text { No tengo en esta la hora. } \\
\text { Enseña á mi la habitacion encima } \\
\text { la azotes. }\end{array}$ \\
\hline $\begin{array}{l}\text { Sube conmigo. } \\
\text { Qué to parece? mira qwe ctare es, }\end{array}$ & $\begin{array}{l}\text { Atla madia. } \\
\text { Ach tqul, chuf kach dhaui, chuf oth- }\end{array}$ & $\begin{array}{l}\text { Sube conmigo. } \\
\text { Qué dices, mira como clara, mira las }\end{array}$ \\
\hline
\end{tabular}

(1) (2) Hesken habitacion en general; bit singular, bius plural, cuartos bajos; rrerfa singulor, rrererf plural, habilaciones principales en el rerandaj $\delta$ galeria; menzeh singular, emnazah plural; habitaciones sobre la azotea. 


\section{Guide}

\section{DIALOGUE XIV.}

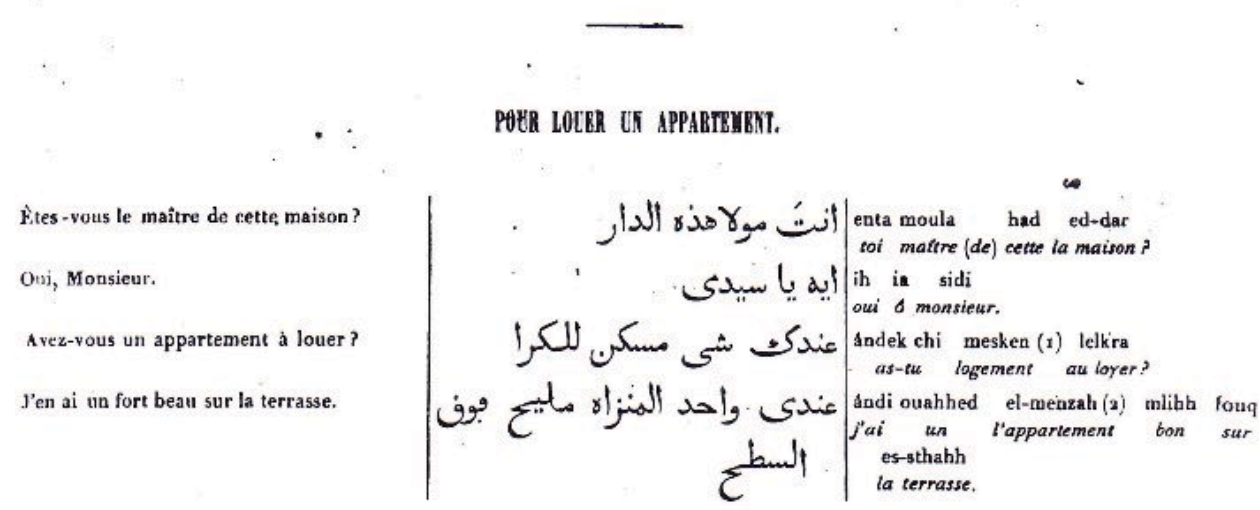

(1-2) On dit aussi بـ bit au sing., بسوت biout au plur., pour désigner une clambre; mais ce mot est particulièremeut usite

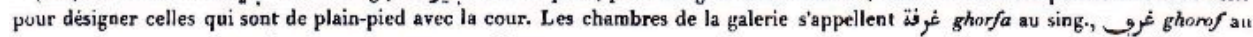
plur.; celles de la terrasse, sfji mensah, au sing., sijo mnazah au plur.

DIA LOGUR XIV.

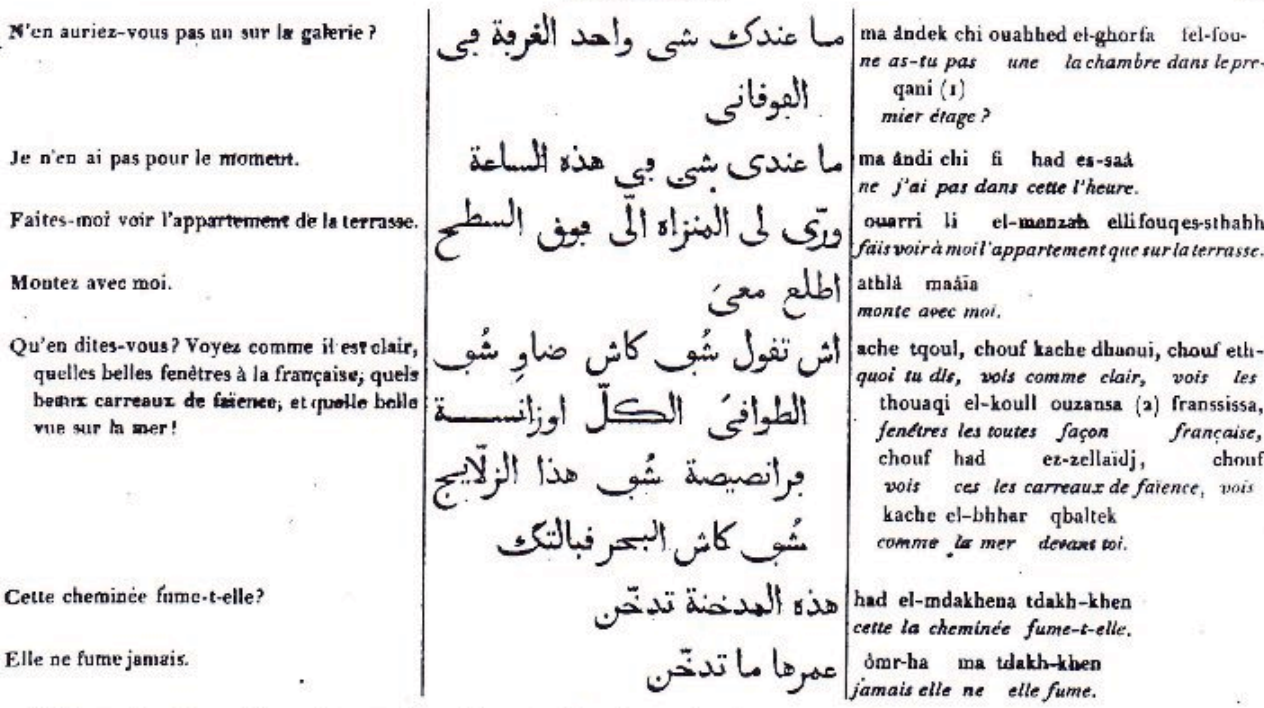

(1) Fouqaai signifie urcobjet: qui esx ux-deasus diun autre Quand ov parle d'une maison a la mauresque, on l'emploie dans le seus de. notre mot premier eloge. - (2). Oasamaa eat emprunté à l'italien usanza. 


\subsection{CAPÍTULO XXI (DIÁLOGOS) Y XXII (GUIDE)}

\section{Diálogos}

\section{DIALOGO XXI.}

\section{Para tomar informes do algana peraona.}

Quién ese señor con quien habla- Achkun hada elli kan itkellem bas hace poco?

Es uno de Tafilete.

Yo crefa que era de Mogador.

Habla muy bien español, francés, inglés, é italiano.

Habla español como un español.

Los franceses to creen francess, Jos ingleses inglés, y los italianos itoliano.

Es muy diffcil poseer bien tantos idiomas.

B. viajado mucho.

Tiene buena memoria. maak del-ueqt chuiia?

Uahed men Thafna.

Tequiasni men Suara.

Itkellem mleh bel sbaniulia, $u$ el françiça, u el enqliza, u eth-thaliana.

Itkellem bel Sbaniulia tequl Sbaajul haqqani.

El-françiç ihasbuh françiç, u el enqliz enqliz, u eth-thalian ihasbuh thalian.

Uaâr elli iâraf hakda el lesun bezzaf.

Safar bezzaf.

Aqlu khefif.

\section{noco?}

Uno de Tafilete.

Pareció á mi do Mogador.

Él babla bien con la española, y la francesa, $y$ la inglesa, y la italiana.

El babla con la española, tú dirás espaĩol verdadero.

Los franceses cucntan á él francés, y los ingleses inglés, y los italianos cuentan á él italiano.

Dificil el que sabe asi las lenguas mucho.

Viajó mucho.

Espíritu suyo ligero.

\section{Guide}

\section{DIALOGUE XXII.}

\section{POTR STIYOBIER OR QTELQOTN,}

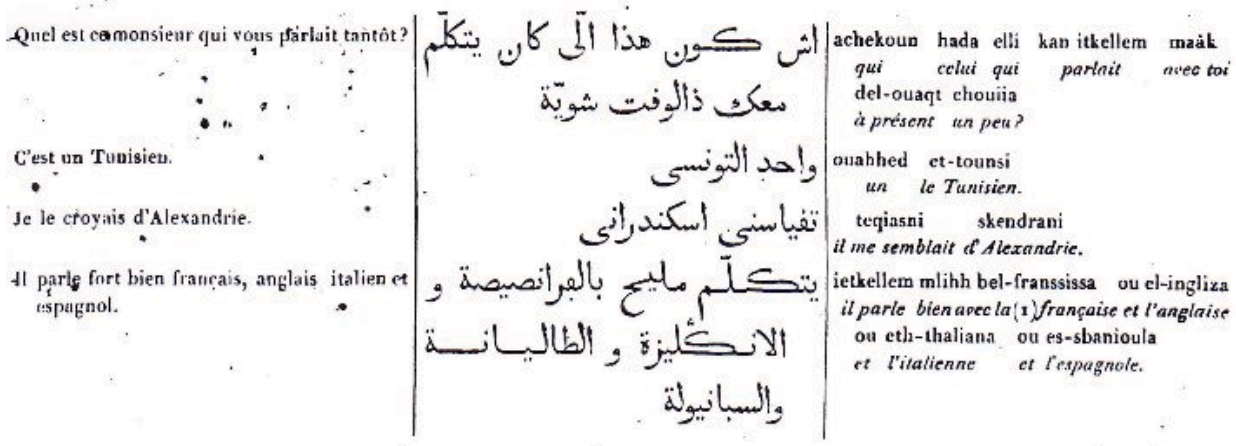

(1) Ici comme plus bas, Isan, langue, est sous-entendu. 
DIALOGUE XXI

Il parle français comme les Français mémes.
II est difticile de posseder bien tant de
Iltalien, et les Espagnols, Espagnol.
langues.

